\title{
Adult smokers' perceptions of cigarette pack inserts promoting cessation: A focus group study
}

\section{Accepted for publication in Tobacco Control published by BMJ Publishing Group.}

\begin{abstract}
Introduction Tobacco companies have a long tradition of including promotional material within cigarette packs, such as cigarette cards and coupons. Only in Canada are they required, by government, to include educational material within cigarette packs, in the form of inserts highlighting the benefits of quitting or providing tips on how to do so.
\end{abstract}

Methods Twenty focus groups were conducted in Glasgow and Edinburgh in 2015 with smokers $(\mathrm{N}=120)$ segmented by age $(16-17,18-24,25-35,36-50,>50)$, gender and social grade, to explore perceptions of the inserts used in Canada.

Results The consensus was that these inserts would capture attention and be read due to their novelty, visibility before reaching the cigarettes, and as they can be removed from the pack. While they may be ignored or discarded, and rotation was considered necessary, they were generally thought to prolong the health message. The positive style of messaging was described as refreshing, educational, encouraging, reassuring and inspirational, and thought to increase message engagement. It was regarded as more sympathetic than command-style messaging, offering smokers 'a bit of hope'. The inserts were often considered preferable to the on-pack warnings, although it was felt that both were needed. Some participants suggested that inserts could encourage them to stop smoking, and they were generally viewed as having the potential to alter the behaviour of others, particularly younger people, would-be smokers and those wanting to quit.

Conclusions Inserts are an inexpensive means of communication and offer regulators a simple way of supplementing on-pack warnings.

\section{INTRODUCTION}

Tobacco companies have used cigarette cards, coupons and inserts for over a century to promote smoking, demonstrating the value that they place on the inside of the cigarette pack. Regulators, in contrast, have almost completely overlooked the potential of using the pack interior to promote quitting. The Canadian government is the exception, having required tobacco companies to include pack inserts since $2000 .{ }^{1}$ The first set of sixteen inserts, designed by the Canadian government to augment the warnings on the pack exterior which were introduced at the same time, was limited to text highlighted in yellow but without full colour, pictures or graphics. ${ }^{2}$ These were replaced in 2012 by eight new inserts with coloured graphics and behavioural recommendations for quitting and highlighting the benefits of doing so, again coinciding with a new set of health warnings. ${ }^{3}$

The reluctance of governments to follow the Canadian lead may be related, in part, to the paucity of research investigating the actual or perceived impacts of inserts. Interviews with smokers in Canada aged 12 to 50 years $(\mathrm{N}=228)$ in 1992 explored their response to inserts with messages about addiction and health risks to smokers and others. ${ }^{4}$ The inserts were generally liked and thought to allow for a lot of information to be presented, with the messages considered believable, important and relevant. ${ }^{4}$ A second interview study in Canada in 2000, with smokers aged 15 years and older $(\mathrm{N}=670)$, explored their perceptions of inserts with messages describing the risks associated with smoking and available help. ${ }^{5}$ Almost half indicated that inserts were a good way to provide information to smokers (48\%), and of those $46 \%$ thought that people would read them. Sixty percent indicated that messages on inserts would be a very effective/somewhat effective way to provide information to smokers who 
want to quit, with approximately two-fifths indicating that they would discourage young people from starting to smoke (42\%) and encourage young smokers to quit (42\%). ${ }^{5}$

Interviews with smokers and recent quitters $(\mathrm{N}=73)$ in New Zealand in 2004 assessed perceptions of warnings and inserts. ${ }^{6}$ Participants were randomly exposed to six inserts, although all were shown an insert used in Canada ('Can second hand smoke harm my family?'). A third (34\%) agreed/strongly agreed that inserts would be an effective way of encouraging reduced consumption or quitting, while a similar proportion (33\%)

disagreed/strongly disagreed. While some were positive about the informative, educational tone of the inserts, the general view was that they would receive only a cursory glance or be ignored completely and would inevitably be discarded. ${ }^{6}$ In the UK, interviews with marketing experts $(\mathrm{N}=12)$ in 2014 explored a number of novel packaging measures, including pack inserts with coloured graphics and either a loss-framed (Smoking damages your lungs) or gain-framed (Quitting will improve your health) message. ${ }^{7}$ Inserts were viewed as another platform for communicating with consumers and a cheap way to capture attention before reaching a cigarette. As on-pack warnings typically feature loss-framed messages, inserts with gain-framed messages highlighting the benefits of quitting (referred to as 'help cards') were thought to be more effective in engaging smokers and more likely to have an impact on those wanting to quit.

Only one study from Canada, a longitudinal online survey with adult smokers between 2012 and 2014, has explored smokers' response to the inserts introduced in 2012.

Approximately a quarter of smokers at each wave reported having read the inserts at least once in the prior month, with younger smokers and those intending to quit or having recently tried to quit significantly more likely to have read them. Smokers who read the inserts a few times or more in the previous month were more likely to have made a quit attempt at the subsequent wave compared to smokers who had not read the inserts. ${ }^{3}$ It was also found that while reading on-pack health warnings significantly decreased across waves, reading inserts significantly increased, with more frequent reading of inserts associated with self-efficacy to quit, quit attempts, and sustained quitting at follow-up. ${ }^{8}$

This study explores the perceptions of adult smokers in Scotland to the inserts with positively-framed cessation messages used in Canada. At the time of the study tobacco packaging within the United Kingdom (Scotland, Wales, Northern Ireland and England) displayed one of two text-only warnings covering $43 \%$ of the pack front and one of 14 pictorial warnings covering 53\% of the pack reverse. These warning messages had been on packs since 2003, required by Tobacco Products Directive 2001/37/EC, ${ }^{9}$ with most (13 of 16) highlighting the negative health risks associated with smoking.

\section{METHODS}

\section{Design and sample}

Twenty focus groups were conducted with smokers $(\mathrm{N}=120)$ in Glasgow and Edinburgh (Scotland) between January and March 2015. Groups were segmented by age (16-17, 18-24, 25-35, 36-50, over 50), gender and social grade (ABC1, C2DE), determined by the occupation of the chief income earner within the household using the National Readership Survey (NRS). The NRS is a well-established classification system in the UK, which categorises social grade into six levels: A, high managerial, administrative, or professional; B, intermediate managerial, administrative, or professional; $\mathrm{C} 1$, skilled non-manual workers; $\mathrm{C} 2$, skilled manual workers; D, semi-skilled and unskilled manual workers; and E, pensioners, casual or lowest grade workers, or unemployed. ${ }^{10}$ Grades A, B and C1 typically signify middle class groups and C2, D and E working class groups. The demographics for each group are shown in Table 1. 
Table 1: Demographic characteristics of, and number within, each group

\begin{tabular}{ccccc}
\hline $\begin{array}{c}\text { Group } \\
\text { number }\end{array}$ & Age group & Gender & Social grade & $\begin{array}{c}\text { Number in } \\
\text { group }\end{array}$ \\
\hline 1 & $16-17$ & Female & ABC1 & 7 \\
2 & $18-24$ & Female & C2DE & 7 \\
3 & $25-35$ & Female & ABC1 & 6 \\
4 & $36-50$ & Female & C2DE & 6 \\
5 & $25-35$ & Female & C2DE & 6 \\
6 & $36-50$ & Female & ABC1 & 5 \\
7 & $18-24$ & Female & ABC1 & 5 \\
8 & $16-17$ & Female & C2DE & 7 \\
9 & $36-50$ & Male & ABC1 & 6 \\
10 & $50+$ & Female & C2DE & 7 \\
11 & $36-50$ & Male & C2DE & 5 \\
12 & $50+$ & Male & C2DE & 6 \\
13 & $50+$ & Female & ABC1 & 6 \\
14 & $50+$ & Male & ABC1 & 7 \\
15 & $25-35$ & Male & C2DE & 6 \\
16 & $18-24$ & Male & ABC1 & 7 \\
17 & $16-17$ & Male & C2DE & 6 \\
18 & $18-24$ & Male & C2DE & 5 \\
19 & $16-17$ & Male & ABC1 & 5 \\
20 & $25-35$ & Male & ABC1 & 5 \\
\hline
\end{tabular}

Participants were recruited in Glasgow and Edinburgh by market researchers using convenience sampling. Market researchers were instructed to intercept potential participants in the street and explain that the study was concerned with smokers' perceptions of tobacco packaging and warnings. A recruitment questionnaire captured information about age, social grade and smoking behaviour. The inclusion criteria were that participants fell within one of the gender, age and social grade groups and smoked cigarettes at least once a week. Response rates were not recorded. Fourteen groups took place in Glasgow, with six in Edinburgh, with each group taking place in a venue suitable for hosting a group discussion (e.g. hotel function room, community centre) and convenient for participants. Venue hire was arranged by the market researchers. Within each group perceptions of inserts and also cigarette design (not discussed here) was assessed.

\section{Procedure}

At recruitment, participants were given an information sheet explaining what the study involved and that it would be audio-recorded, and asked to complete a consent form. The information sheet and consent form explained that the study was anonymous and confidential and that participants could withdraw at any time and did not have to respond to any questions. The consent form also stated that all materials, including audio recordings, would be stored securely at the University of Stirling. At the start of each group participants were reminded of confidentiality, anonymity, their right to withdraw and not to respond to questions, and also informed that their views may differ and when answering they should not be influenced by anyone else within the group or the interviewer(s). At the end of each group, which typically 
lasted ninety minutes, participants were debriefed about the purpose of the study, and received a $£ 25$ incentive fee.

All groups were moderated by one or more experienced facilitators employed by the University of Stirling. No-one other than the interviewer(s) and participants, none of whom were known to the interviewer(s), were present during the groups. A discussion guide was used to ensure that common questions were asked across groups, but otherwise participants were allowed to lead the discussion on inserts, with the interviewer(s) following up on comments made. Participants were informed that in Canada every cigarette pack contains an insert, with a total of eight rotating inserts used. Each group was then shown two inserts considered relevant to their age and gender, with seven inserts used. Young adult males (1617, 18-24, 25-35) were shown an insert explaining the benefits of quitting (Quitting... What's in it for me?) and an insert about cravings (They only last an average of a few minutes). Young adult females were shown an insert explaining the benefits of quitting before pregnancy (Thinking of having a baby?), with 16-17 and 18-24 year olds also shown the insert explaining the benefits of quitting and 25-35 year olds the insert about cravings. Those aged 36-50 years were shown an insert encouraging smokers to 'Never quit trying to quit', with females also shown an insert about quitting (I had enough of feeling guilty) and males the insert about cravings. Groups over the age of 50 were shown an insert about 'Morning cough' and an insert explaining that 'It's never too late' to quit (the seven inserts used are shown in Figure 1). Groups were given time to look at the inserts and asked whether they thought they would read them, what their perceptions of them were, and whether they may be helpful to them and others, e.g. 'Do you think that you would read these inserts if they appeared in your pack?'

Figure 1: The seven inserts used in the study

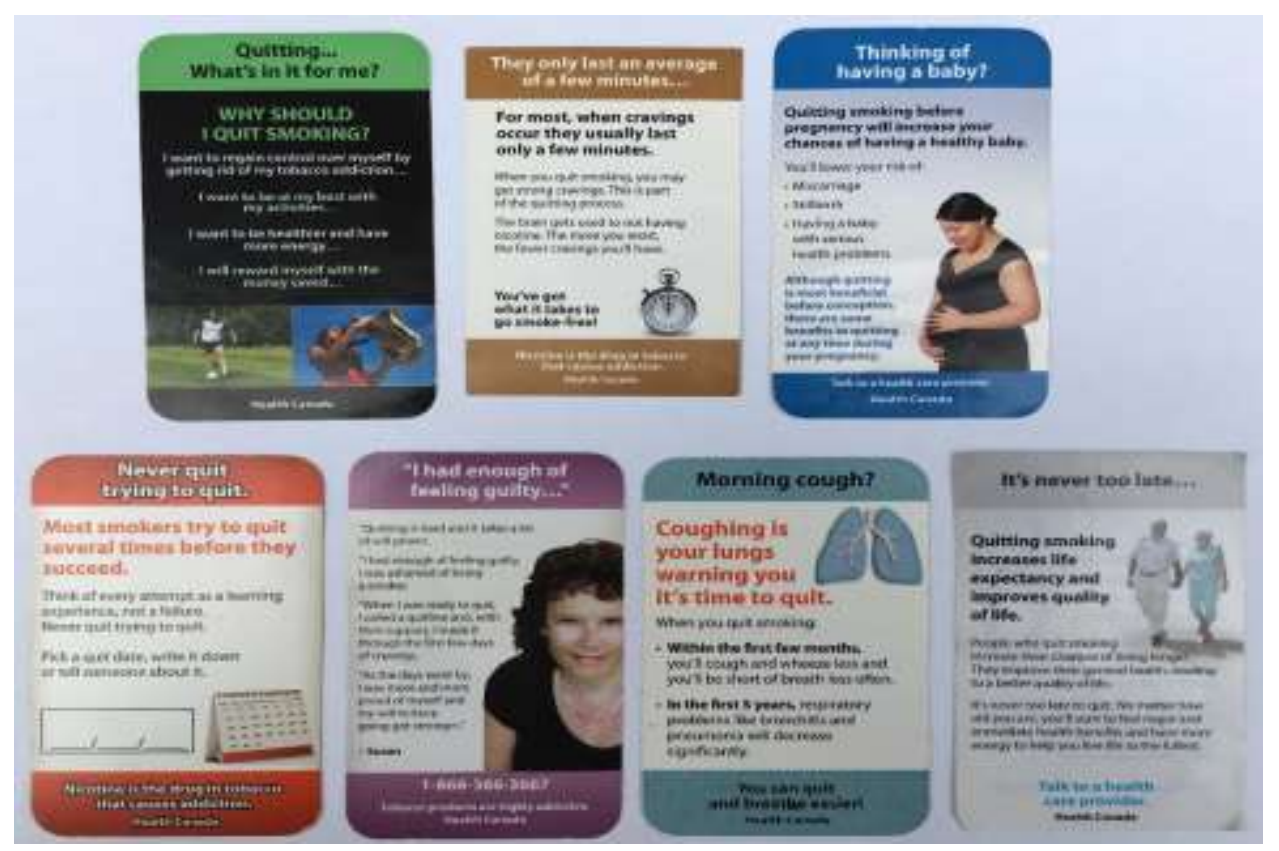

\section{Analysis}

The author checked the transcripts against the audio files to ensure accuracy and then reviewed them using an iterative approach, categorising content according to inductively developed thematic codes pertaining to specific quotations and relevant text. Each transcript was then re-assessed to identify any relevant quotes, originally missed, that could add to any 
of the four main themes identified (Salience, Message style, Supplement on-pack warnings, Perceived impact on self and others).

\section{RESULTS}

\section{Salience}

The consensus was that inserts would capture attention and be read, at least once, because of their novelty and their visibility before reaching the cigarettes. The different colours used on the inserts were also thought by some younger males to draw attention to them.

I know what everybody's saying, aye, definitely you're going to read it because it's something that you've no seen before (25-35 Female, C2DE)

What's good about that is you have to pull that out to get to your cigarettes, so you can't escape it (18-24 Male, ABC1)

Participants in several groups, more frequently males and those from a lower social grade, commented that inserts would be discarded or ignored, e.g. 'I'm an ardent smoker, I'd just crunch it up and throw it away' (25-35 Male, C2DE). However, in general they were thought to prolong the message. Unlike the warnings on packs, which are discarded after use, it was felt that the inserts would have a longer lasting impact as they would be removed from the pack and remain within the household or elsewhere, or be visible as litter.

It's something that would fall out of your packet and sit about your house for a while, so you would see it more. You chuck out your packet as soon as it's done (18-24 Female, C2DE)

They'll get left about on tables and other people will pick them up and sit and read it. I think these inserts will end up everywhere and people will pick them up to see what it is (50+ Male, C2DE)

Several participants suggested that they would intentionally retain the inserts, for instance in their pocket or purse, particularly if attempting to quit.

The chance of being infertile because I've smoked does actually scare me so something that's got information, has got somewhere you can go, call or visit online, would be, you could fit that in your purse so you'd be more inclined to keep it (25-35 Female, ABC1)

If I had opened my packet, looked at those words and decided I was quitting and phoned the number and thought right - I would keep that. See when I wanted a fag, I would read that one, that is an inspirational one (36-50 Female, ABC1)

Message style

Groups were shown inserts considered relevant to their age, which were generally considered relatable. For instance, one of the inserts used with younger age groups, which showed a male playing football and a female playing the saxophone and explaining that quitting allows people to be the best at their activities, have more energy, and be able to reward themselves with the money saved, was considered relevant, even if the image of the female playing the saxophone was mocked by some participants as it was not considered realistic (see Figure 1). 
The three benefits - it's something that we've all said, so it relates to you. (She) said it was affecting her health and money, the shoes you want to buy. It ties in to everyone (16-17 Female, C2DE)

I can kind of relate to that activity one, in a way, cause usually I play football on a Monday night and it's usually the worst time. I'll be running round and after ten minutes (makes gasping sound) think I maybe shouldn't of had so many over the weekend (18-24 Male, ABC1)

The positive messaging was consistently well-received, although in one group of young women smokers the insert explaining that quitting smoking before pregnancy would increase the chances of having a healthy baby was considered 'patronising', 'annoying' and 'offensive' (18-24 Female, C2DE). This group aside, the messaging was described as refreshing, educational, informative, helpful, encouraging, reassuring and motivational, and considered to increase message engagement. It was viewed as a more sympathetic approach than command-style messaging, similar to advice or a gentle reminder, e.g. 'That is something I already know but it's like a gentle reminder and there may just be a day where I'll click' (36-50 Female, C2DE). In several mostly female groups participants commented that the inserts offered 'a bit of hope' (50+ Female, ABC1).

I think it's just helpful for anyone willing to read it, Helpful facts, Good facts to know about smoking (16-17 Male, C2DE)

It's a softer approach and it is more understanding. It doesn't poke you in the eye and try to make you feel bad about yourself (36-50 Male, ABC1)

While it was explained that there were a total of eight inserts, rotation was considered necessary to prevent desensitisation, as with the on-pack warnings.

If there was lots of them, you'd maybe be more inclined to read them (25-35 Female, $\mathrm{ABC} 1)$

They'd need to have a rotation, aye, cause you just get used to it...

You'd get used to it

... and just blank it out (36-50 Female, C2DE)

\section{Supplement on-pack warnings}

As a result of the style of messaging used on the inserts they were often preferred to on-pack health warnings, which were generally disliked. Several mostly female groups however felt that to maximise impact there was a need for both hard-hitting warnings and positive messaging, e.g. 'Shocking backed up with a wee bit of positive, it would probably have more effect' (36-50 Female, ABC1).

When you see the front of the pack and you're having a think about it and saying what am I doing to myself and then you open it up and see a card inside, like, this is what it could be like if you quit, then I guess it works well together (16-17 Female, C2DE) 
It might be quite good to have something like that as well as they horrible pictures so then you've actually got something informative (25-35 Female, ABC1)

\section{Perceived impact on self and others}

In several groups participants suggested that inserts may make them think about or question their smoking behaviour and encourage them to stop. That the inserts highlighted the positives of stopping, and some offered tips on how to quit, increased the feeling of optimism about quitting for some.

They make you think more about quitting. When you open it, you'd see it. Instead of, when you've got the packet, you don't take any notice of it but when you'd open it, you'd actually take it out and see it and think about it.

I think it encourages you to stop smoking (16-17 Female, ABC1)

It gives you a bit more motivation to stop.

\section{A bit of motivation? (moderator)}

Aye, because that is when you read that, you are reading yourself that question, asking yourself that question, why should I quit smoking, what will I benefit from it? (18-24 Male, C2DE)

It gives you a bit of hope, look at the benefits. I'm going to get lung cancer, I'm going to get this, I'm going to die, maybe not looking at this. I should stop (50+ Female, $\mathrm{ABC} 1)$

There was also mention of smokers potentially being influenced by significant others who may be exposed to the inserts, for instance children or partners.

Again that having come out of a packet and if it does get left on a table and as you said, it is quite durable - a kid can pick that up from the table and say, dad, why are you not getting a date on this? So there is maybe a wee bit more pressure coming from external sources. I think that might be a shove in the right direction (36-50 Male, $\mathrm{ABC} 1)$.

If your partner, he read the pregnancy one, he might coax you (to stop) (25-35 Female, $\mathrm{ABC} 1)$

Perceived behavioural impact was thought to be influenced by multiple factors. As mentioned within several groups, a desire to quit and willpower were considered pre-requisites. Within predominantly middle (36-50) and older age groups (50+) comments were made that the inserts would be of little use given prior awareness of the information, the perceived difficulty overcoming addiction, or a sense that it was too late to change.

There comes a point in people's lives where they think yeah this is it, I don't want to smoke anymore. I think you have to really want it, I don't think it would just come from reading a message. Maybe over a period of time, if you read those messages over and over again, it would filter in (18-24 Female, ABC1)

It's too late for us

That's it, it's too late (50+ Female, C2DE) 
In terms of the impact on others, inserts were thought to be of value for smokers thinking about quitting and females that are or plan to become pregnant. Several groups aged 25 or over suggested that inserts had the potential to influence the behaviour of younger people, while several lower social grade groups under the age of 25 commented that they could be helpful for would-be smokers.

It might no help us but generations further down it's going to help (25-35 Female, C2DE)

If someone says, oh can I have a fag and you know that they don't smoke you could be like...

Have a wee read of the card

...read this first and if you are still sure that you want a fag then have one (16-17

Male, C2DE)

\section{DISCUSSION}

That pack inserts are an established form of marketing, ${ }^{11}$ an inexpensive means of reaching smokers, ${ }^{11}$ and offer almost unlimited opportunities for communication, ${ }^{2}$ helps to explain why they are so frequently used by tobacco companies. It also raises questions about why they have been so infrequently explored, or even discussed, within tobacco control.

The inserts were thought to capture attention due to their novelty, visibility when taking a cigarette and because they could be removed from the pack. In previous research marketing experts expressed a similar view, equating an insert to a door that a smoker would have to go through to reach a cigarette, thus providing information at the 'decision moment'. While it was commented, particularly among males and those from a lower social grade, that inserts could be ignored and discarded (consistent with previous research in New Zealand ${ }^{6}$ ), it was felt that they would have a longer lasting impact than the on-pack health warnings because after being removed from packs they may remain within the household or be retained by the smoker. This would also apply to cigarette cards, coupons and promotional inserts, and this extended communication may explain why they have been a mainstay of tobacco marketing. ${ }^{12-15}$

It has been argued, for at least thirty years, that tobacco companies' duty to inform requires both on-pack warnings and pack inserts, as often used by manufacturers of medications and other consumer products. ${ }^{16}$ Early research on inserts however failed to explore how they compare to, or would complement, on-pack warnings. ${ }^{4-6}$ In a recent study with marketing experts, the general view was that smokers would not like, read or engage with inserts with negatively-framed messages as this is the same style of messaging used on the on-pack warnings, but they would respond to inserts with positively-framed messaging. ${ }^{7}$ Similarly, in this study the inserts were thought to supplement the on-pack warnings because of the contrasting styles of messaging, with the on-pack warnings highlighting the negatives of continuing to smoke and the pack inserts explicating the positives of quitting. It is common for a set of on-pack warnings to comprise mostly fear-based messages, which are more likely to be recalled in population-based surveys than positive-themed cessation messages and typically rated as having a greater impact, ${ }^{17}$ with cessation messages on the pack exterior of limited value for non-smokers. Once the pack has been purchased and opened however positive cessation messaging becomes highly relevant, and there is evidence that such messages may be more effective in encouraging adult smokers to quit. ${ }^{18-20}$

Some participants indicated that the inserts could influence how they think about their own smoking behaviour, due to the positive messaging and tips on cessation, with possible pressure from significant others (e.g. children) exposed to the inserts. It was felt that inserts 
would be most effective for younger people and would-be smokers, and also those wanting to quit and pregnant women. This is consistent with past research, which has found that inserts would be most likely to have an impact on those wanting to quit, ${ }^{3,7}$ and may help deter young people from starting or continuing to smoke. ${ }^{5}$ While some participants considered inserts to be annoying or of negligible value for various reasons (e.g. prior awareness of the information presented or perceived difficulty in overcoming addiction), particularly middle- and olderaged participants, notably there was little opposition to their inclusion in packs. However, as the study did not directly explore how supportive smokers are of inserts encouraging cessation, future research doing so would be fruitful.

In terms of limitations, although the sample consisted of young, middle and older aged adult male and female smokers from different socioeconomic backgrounds, the views expressed are not necessarily representative of the wider population of smokers. It is also possible that the generally positive perceptions of the inserts may be due to their novelty or the fact that groups were only shown two inserts relevant to them, rather than the full set of eight used in Canada which includes inserts that groups may not consider relatable. In addition, the study only provides an insight into the perceived (rather than actual) impacts of inserts. Additional research from Canada exploring the longer term impacts of inserts, including wear-out, or the role of significant others on smokers' response to inserts, would be helpful. Studies from other countries using naturalistic designs to explore smokers' response to inserts as they go about their daily lives, would also be of value; naturalistic designs have previously been employed to explore plain packaging and pictorial health warnings. ${ }^{21-4}$

While fear-based warnings can create high threat, it is argued that this 'shock, horror' approach is most effective if supported by more optimistic messaging, ${ }^{25,26}$ consistent with the health communication literature. ${ }^{27}$ For tobacco products, fear-based on-pack warnings are the global norm and likely to remain so. For instance, within Europe a new Tobacco Products Directive (2014/40/EC), ${ }^{28}$ which applies to all 28 European Union (EU) member Parties but will also be adopted by several non-EU European countries, requires 14 rotating pictorial warnings covering $65 \%$ of the front and back of cigarette packs from 2017 , with all but one of these warnings fear-based. What is generally missing or less prominent is messaging intended to provide smokers with a belief that they are capable of quitting. Inserts explaining the benefits of quitting and promoting self-efficacy to do so would appear to be an appropriate complement to the on-pack warnings.

\section{What this paper adds}

- This study explores Scottish smokers' response to pack inserts used in Canada, which feature coloured graphics and messages highlighting the benefits of quitting or providing tips on how to do so.

- The inserts were thought to capture attention, increase message engagement, supplement the on-pack warnings, prolong the health message, and potentially influence the behaviour of younger people, would-be smokers and smokers wanting to quit.

- Inserts are a simple and inexpensive means of communicating with smokers and a viable option for regulators.

Acknowledgements The author would like to thank Jennifer McKell, Richard Purves and Fiona Dobbie for helping to conduct the groups, and David Hammond for providing the inserts.

Funding This work was supported by a grant from Cancer Research UK (A16943).

Competing interests None declared. 
Ethical approval Granted by Ethics Committee of the School of Management, University of Stirling.

Provenance and peer review Not commissioned; externally peer reviewed.

\section{REFERENCES}

1 Health Canada. Regulatory impact analysis statement (Tobacco Products Information Regulations). Canada Gazette 2000;134:1738-848.

2 Mahood G. Canada's tobacco package label or warning system: "telling the truth" about tobacco product risks. Geneva: WHO; 2004.

3 Thrasher JT, Osman A, Abad EN, et al. The innovative use of cigarette package inserts to supplement pictorial health warnings: An evaluation of the Canadian policy. Nic Tob Res 2015;17:870-5.

4 Tandemar Research Inc. Tobacco health warning messages, inserts and toxic constituents information study - Final Report. Toronto: Tandemar Research Inc; 1992.

5 Environics. Health warning messages on the flip/side and inserts of cigarette packaging. A survey of smokers. A report prepared for Health Canada; 2000.

6 BRC Marketing and Social Research. Smoking health warnings study. Wellington: Ministry of Health; 2004.

7 Moodie C. Novel ways of using tobacco packaging to communicate health messages: Interviews with packaging and marketing experts. Addiction Res Theory 2016;24:5461.

8 Thrasher JF, Swayampakala K, Cummings KM, et al. Cigarette package inserts can promote efficacy beliefs and sustained smoking cessation attempts: A longitudinal assessment of an innovative policy in Canada. Prev Med 2016;88:59-65.

9 EU Directive 2001/37/EC of the European Parliament and of the Council of 5th June 2001. Official Journal of the European Communities, 2001.

10 Grant A, Morrison R, Dockrell MJ. Prevalence of waterpipe (shisha, narghille, hookah) use among adults in Great Britain and factors associated with waterpipe use: data from cross-sectional online surveys in 2012 and 2013. Nic Tob Res 2014;16:9318 .

11 Rowse EJ, Fish LJ. Fundamentals of advertising. Whitefish, MT: Kessinger Publishing; 2005.

12 London Cigarette Company. The catalogue of international cigarette cards. Exeter, England: Webb \& Bower; 1982.

13 Lee YJ. The study and conservation of woven cigarette inserts. J Korean Society Clothing Textiles 2006;30:447-57.

14 Simpson D. USA/Brazil: the flavour of things to come? Tob Control 2004;13:105-6. 
15 Moodie C, Hastings GB. Tobacco packaging as promotion. Tob Control 2010;19:16870 .

16 McLeod K. The Great American Smokeout: Holding cigarette manufacturers liable for failing to provide adequate warnings of the hazards of smoking. Boston Coll Law Rev 1986:27:1033-74.

17 Hammond D. Health warning messages on tobacco products: a review. Tob Control 2011;20:327-37.

18 Gallagher KM, Updegraff JA. Health message framing effects on attitudes, intentions and behaviour: A meta-analytic review. Ann Behav Med 2011;43:101-16.

19 Toll BA, Rojewski AM, Duncan LR, et al. "Quitting smoking will benefit your health": The evolution of clinician messaging to encourage tobacco cessation. Clin Cancer Res 2014;20:301-9.

20 Cataldo JK, Hunter J, Petersen AB, et al. Positive and instructive anti-smoking messages speak to older smokers: A focus group study. Tob Induc Dis 2015;13:2.

21 Moodie C, Hastings GB, Mackintosh AM, et al. Young adult smokers' perceptions of plain packaging: A pilot naturalistic study. Tob Control 2011;20:367-73.

22 Moodie C, Mackintosh AM. Young adult women smokers' perceptions of using plain cigarette packaging: A naturalistic approach. Br Med J Open 2013;3:e002402.

23 Mays D, Murphy SE, Johnson AC. A pilot study of research methods for determining the impact of pictorial cigarette warning labels among smokers. Tob Induced Diseases 2014;12:16.

24 Gallopel-Morvan K, Moodie C, Eker F, et al. Perceptions of plain packaging among young adult rollyour- own smokers in France: A naturalistic approach. Tob Control 2015;24:e39-44.

25 White CJ, Thomas H. Up in smoke: demarketing and consumer smoking cessation. Int J Consumer Studies 2016;40:75-82

26 Mead EL, Cohen JE, Kennedy CE, et al. The role of theory-driven graphic warning labels in motivation to quit: a qualitative study on perceptions from low-income, urban smokers. BMC Pub Health 2015;15:92.

27 Maloney EK, Lapinski KL, Witte K. Fear appeals and persuasion: A review and update of the Extended Parallel Process Model. Soc Personal Psychol Compass 2011;5/4:206-19.

28 EU Directive 2014/40/EC of the European Parliament and of the Council of 3rd April 2014. Official Journal of the European Communities, 2014. 